
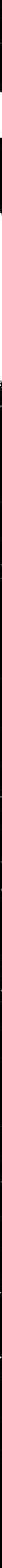

in

$1-$
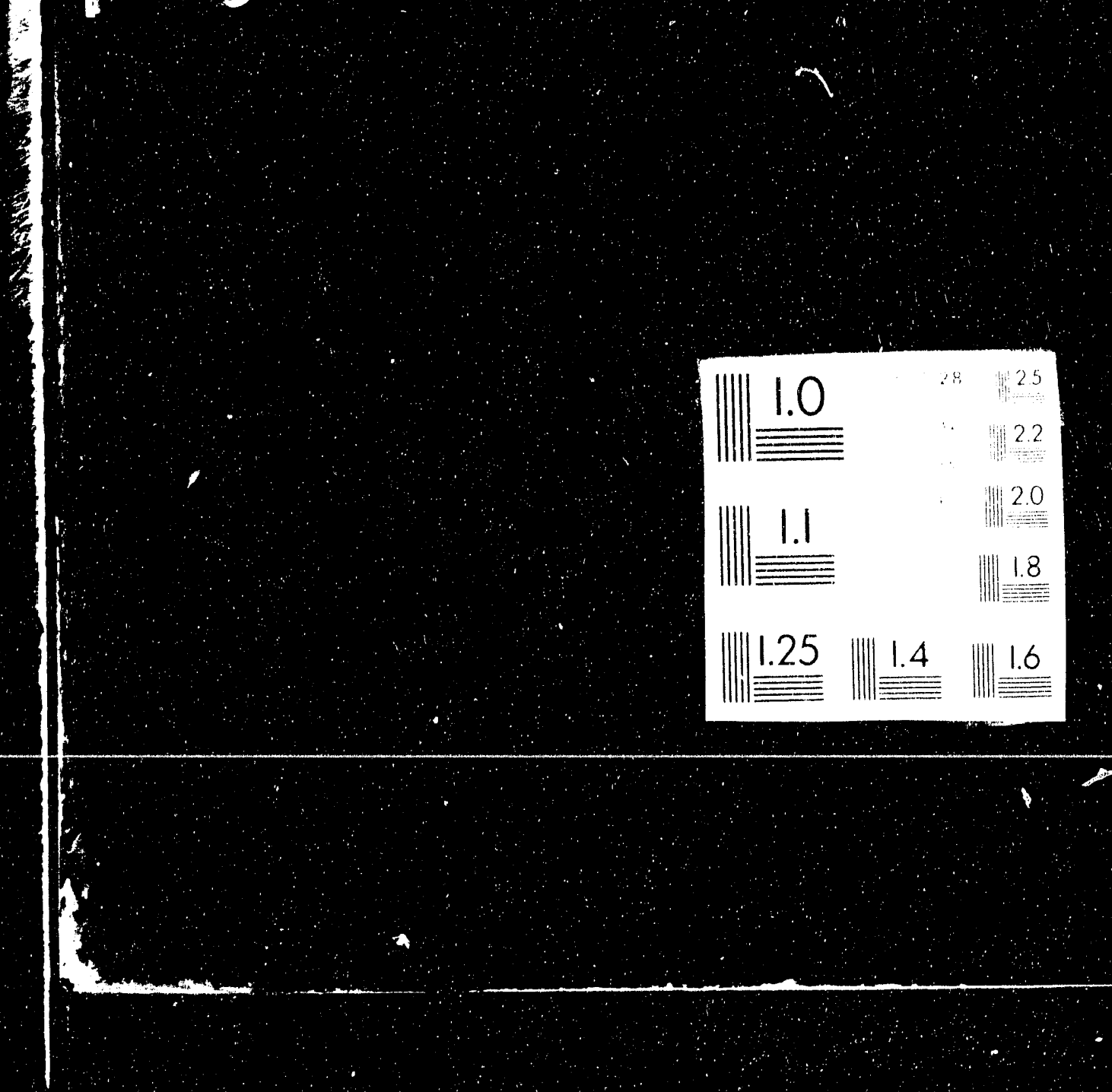


\title{
Advanced Computational Methods for Nodal Diffusion, Monte Carlo, and SN Problems
}

\author{
Progress Report for the Period \\ January 1, 1992 - March 31, 1993

\section{Project Director} \\ Edward W. Larsen

\section{Co-principal Investigator} \\ William R. Martin

\section{Research Assistants} \\ Kasem Abotel \\ Amitava Majurndar \\ Moaied Miften \\ Dorde Tomasevic \\ Scott Turner \\ Department of Nuclear Engineering \\ The University of Michigan \\ Ann Arbor, Michigan 48109-2104
}

DOE/ER/75709--1

DE93 006816

January, 1993

\begin{abstract}
This report was prepared as an account of work sponsored by the United States Government. Neither the United States nor the Department of Energy, nor any of their employees, nor any of their contractors, subcontractors, or their employees, makes any warranty, express or implied, or assumes any legal liability or responsibility for the accuracy, completeness, or usefulness of any information, apparatus, product or process disclosed or represents that its use would not infringe privately-owned rights.
\end{abstract}

\section{Prepared for}

THE U. S. DEPARTMENT OF ENERGY

GRANT NO. DE-FG02-92ER-75709 


\begin{abstract}
This proposal describes progress on five separate research efforts for improving the effectiveness of computational methods for particle diffusion and transport problems. These are:

- The development of multigrid methods for obtaining rapidly converging solutions of nodal diffusion problems. A alternative line relaxation scheme has been found to be promising and is being implemented into a nodal diffusion code. In addition, simplified P2 has been implemented into this code with excellent results compared to transport calculations.

- The development of the Local Exponential Transform method for variance reduction in Monte Carlo neutron transport calculations. This work has been very successful, yielding predictions for both 1-D and 2-D x-y geometry which are significantly better than conventional Monte Carlo with splitting and Russian Roulette.

- The implementation of Asymptotic Diffusion Synthetic Acceleration methods for obtaining accurate and rapidly converging solutions of certain multidimensional $S_{N}$ problems. New transport differencing schemes have been obtained that allow solution by the conjugate gradient method, and the convergence of this approach is rapid.

- The development of Quasidiffusion (QD) methods for obtaining accurate and rapidly converging solutions of multidimensional $S_{N}$ problems on irregular spatial grids. A "symmetrized" QD method has been developed and implemented in a form that results in a system of two self-adjoint equations that are readily discretized and efficiently solved.

- The implementation of the response history method for speeding up the Monte Carlo calculation of electron transport problems. Although this effort was not funded during the first year of the project, we have been able to implement the response history method into the MCNP Monte Carlo code. In addition, we have developed and implemented a parallel time-dependent Monte Carlo code on two massively parallel processors.
\end{abstract}

Progress on these projects has greatly enhanced our ability to obtain accurate and efficient solutions for many diffusion and transport problems encountered in nuclear engineering applications. 


\section{Table of Contents}

1. Introduction and Objectives........................................................................... 4

2. Progress Report for the Period 1/1/92 - 3/31/93 ............................................ 5

A. Nodal Diffusion: Multigrid Algorithms .............................................. 5

B. Monte Carlo: Local Exponential Transform......................................... 7

C. Deterministic $S_{N}$ : DSA Acceleration ............................................... 12

D. Deterministic $S_{N}$ : Quasidiffusion Transport Algorithms...................... 14

E. Monte Carlo: Response History Method ............................................ 19

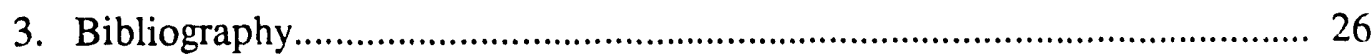




\section{Introduction and Objectives}

This research project includes effort in several areas related to computational particle transport, including nodal diffusion methods, Monte Carlo methods, and deterministic transport methods, such as discrete ordinates $\left(\mathrm{S}_{\mathrm{N}}\right)$ methods. As given in the original University of Michigan (UM) proposal to the Department of Energy (DOE) in January 1991, the UM proposed to address the following specific topics in computational particle transport:

Task 1. Nodal diffusion: multigrid algorithms. The development of multigrid methods for obtaining rapidly-converging solutions of nodal diffusion problems.

Task 2. Monte Carlo: Local exponential transform. The development of the Local Exponential Transform method for variance reduction in Monte Carlo neutron transport calculations.

Task 3. Deterministic Transport: DSA Acceleration. The implementation of Asymptotic Diffusion Synthetic Acceleration (DSA) methods for obtaining accurate and rapidly converging solutions of certain multidimensional $S_{N}$ problems.

Task 4. Deterministic Transport: Quasi-diffusion methods. The development of Quasidiffusion methods for obtaining accurate and rapidly converging solutions of multidimensional $S_{\mathrm{N}}$ problems on irregular spatial grids.

Task 5. Monte Carlo: Response History Method and MPPs. The development and implementation of a method similar to the response matrix nsethod for deterministic particle transport, for applications to electron transport problems.

Originally, Task 5 appeared as Task 2 in the UM proposal, and the other tasks were appropriately renumbered, but it is included here as Task 5 to reflect the fact that it is an additional task that was not supported by this contract. We are proposing to include it in the renewal contract. Since we were able to find internal UM support and other external support for limited work on this task, progress on this task will be included in this report. In addition, we have devoted effort to utilize massively parallel processors (MPPs) for Monte Carlo particle transport, a subject consistent with the overall technical scope of the research being supported by this project. The reason for this change in direction for our Monte Carlo effort was the acquisition by the UM of a state-of-the-art MPP in June 1992, and we decided to take advantage of this opportunity to direct our efforts towards utilizing MPPs for computational particle transport. We are also proposing to continue this MPP effort for the renewal contract. 


\section{Progress Report for the Period 1/1/92 - 3/31/93}

\section{A. Nodal Diffusion and Simplified $\mathrm{P}_{\mathrm{N}}$ Methods}

One of the most significant developments of the last twenty years has been the advent of nodal diffusion methods for nuclear engineering problems [1-3]. Briefly, nodal diffusion methods are a procedure for replacing a single multidimensional diffusion equation by a system of coupled one-dimensional equations which is solved exactly - except for certain approximations that are introduced into the transverse-leakage terms. Nodal methods have become widely-used because of their ability to produce very accurate solutions to problems on relatively coarse meshes. However, the iterative procedures that have been used for solving the discretized nodal diffusion equations become inefficient for large physical systems with small absorption cross sections. To remedy this difficulty, we have proposed to develop multigrid algorithms [4-6] for nodal diffusion equations.

Nodal diffusion methods are usually formulated with unknowns located on the edges of the spatial grid. Two types of equations are usually obtained, each with seven unknowns (in the simplest flat leakage approximation), one (a) spanning an array of $2 \times 1$ cells, the other (b) spanning an array of $1 \times 2$ cells. [See Fig. 1.]

Figure 1: Nodal Diffusion Method Templates

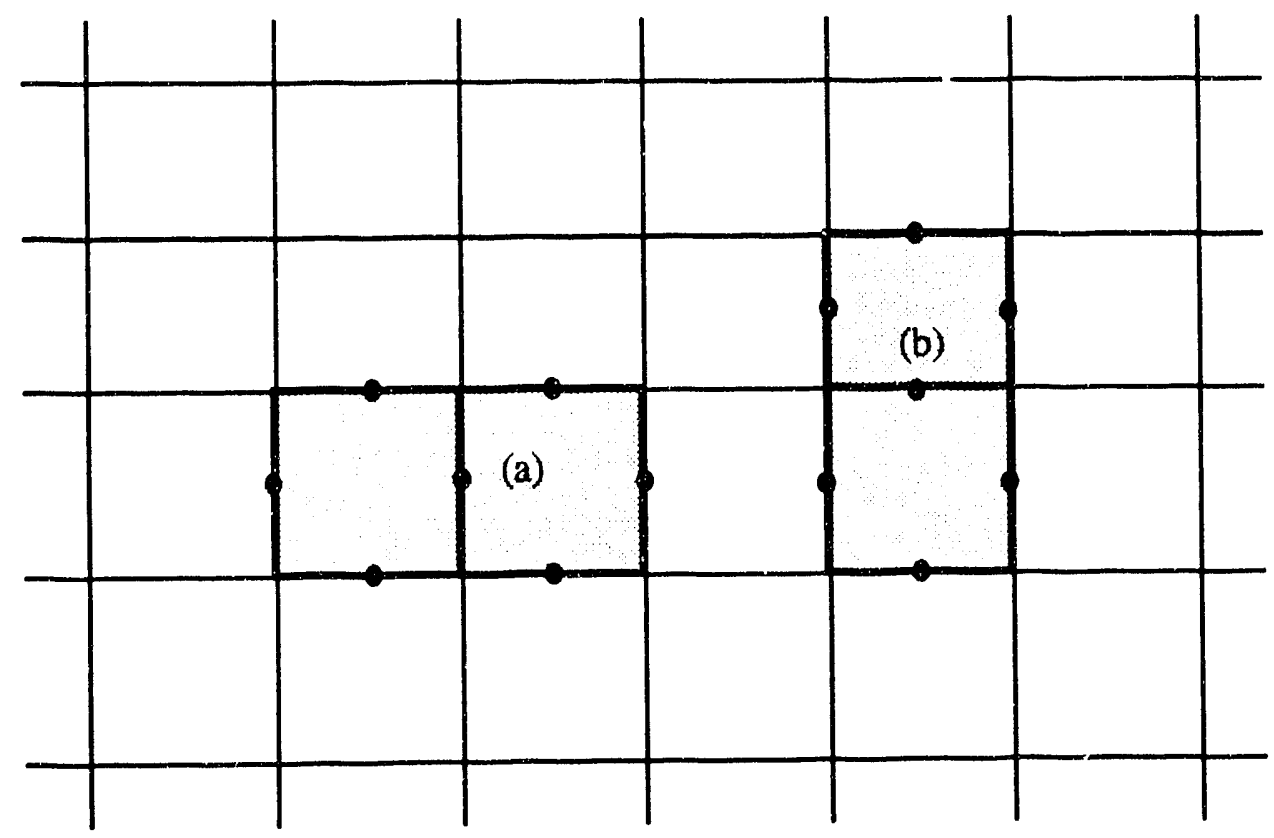


In our original proposal, we outlined an approach termed "four-cell block inversions," which we had hoped would form the basis of an efficient "smoother" for a multigrid algorithm. However, a Fourier analysis of this concept has shown that it will not work. Instead, we have determined theoretically (using a Fourier analysis) that a form of line relaxation can be used efficiently. The basic idea is described as follows.

First, let us "freeze" all of the horizontal cell-edge unknowns and update the vertical celledge unknowns using the templates labeled (a) in Figure 1. This can be done by solving independent tridiagonal systems of equations, one system for each row in the original spatial grid. Next, let us "freeze" all of the vertical cell-edge unknowns and update the horizontal cell-edge unknowns using the templates labeled (b) in Figure 1. This can be done by solving independent tridiagonal systems of equations, one system for each column in the original spatial grid. We are now in the process of implementing a variation of this method in a special test code, CASTOR.

In addition, we have done a considerable amount of work to implement several known high-order nodal diffusion methods into CASTOR. The differences between these methods depend on the order of the (polynomial) approximations used to approximate the transverse leakage terms. The code now permits multigroup problems to be solved with substantially high-order polynomial approximations to the transverse leakage terms. This will make the code a very useful vehicle for the testing of multigrid methods.

In related work, we have developed a variational theory for for deriving slab-geometry $\mathrm{P}_{2}$ equations with boundary conditions [7], and an asymptotic theory for deriving the simplified $\mathrm{P}_{\mathrm{N}}$ $\left(\mathrm{SP}_{\mathrm{N}}\right)$ equations in general geometry $[8,9]$. These theoretical advances have been accompanied with numerical results, which show that the new theories have substantial advantages over diffusion theory. Specifically, the slab-geometry $\mathrm{P}_{2}$ equations with variationally-derived boundary conditions are shown to be nearly always more accurate than standard diffusion theory, and in 3-D calculations, the $\mathrm{SP}_{3}$ equations are also shown to be significantly more accurate than diffusion theory.

As a consequence of this work, we have adapted our nodal diffusion code CASTOR to solve $x, y$-geometry $\mathrm{SP}_{2}$ calculations, and we have shown that such calculations are nearly always much closer to the transport calculation than the $\mathrm{P}_{1}$ solution [10]. In fact, $\mathrm{SP}_{2}$ problems can be formulated exactly into the form of a $\mathrm{P}_{1}$ problem, with modified definitions of the unknowns and cross sections. Therefore, the numerical implementation of $\mathrm{SP}_{2}$ problems effectively requires only that one have an accurate and efficient diffusion solver. 


\section{B. Monte Carlo: Local Exponential Transform}

The conventional Exponential Transform method [11-13] is a well-known but little-used variance reduction method for Monte Carlo source-response calculations. The reason why the method is little-used is that the programmer must input parameters which bias particles to travel preferentially in certain directions. The optimal definition of these parameters is not known a priori, and if a sufficiently incorrect choice is made, the resulting calculation can have a larger variance than the analog calculation. In fact, in some cases, the resulting variance can even be infinite. Therefore, the method is "dangerous" in the sense that the variance of the solution can depend sensitively on parameters that must be chosen by the programmer.

For source-detector problems, we have formulated a zero-variance method, which has the property that every source particle is guaranteed to eventually score, and when it does, it has exactly the correct weight. In principle, using such a method, one would need only one particle to obtain the correct solution. However, this zero-variance method has the deficiency that in order to implement it, one must have complete and exact knowledge of the solution of an adjoint transport problem. Having this adjoint solution, one can formulate modified cross sections which govern the transport process satisfied by the zero-variance particles. However, in practice, it is just as difficult to solve the adjoint transport problem as it is to solve the original forward transport problem.

Using the impractical zero-variance method as a guide, we have developed and implemented an approximate but practical version of this method, which we have termed the Local Exponential Transform (LET) method [14,15]. In the LET method, we first solve numerically the (multigroup) diffusion approximation to the adjoint transport problem that is necessary for the zero variance method. Then, within each cell, the spatial variation of each adjoint transport group flux is approximated by a (spatially) exponential function, with group-dependent amplitudes and decay constants (the biasing parameters) determined by the numerical diffusion solution. The decay constants have vector properties in multidimensional geometry problems. The direction of the vector represents the preferred direction of biasing and the amplitude of the vector represents the strength of the biasing. If the approximation to the adjoint transport solution is taken to be isotropic in angle, we obtain a Monte Carlo variance reduction method with no angular biasing. If the approximation has angular dependence, we obtain a Monte Carlo variance reduction method with angular biasing. In either case, the implementation of the method in each spatial cell very closely resembles that of the conventional Exponential Transform method. However, the key difference between the conventional and "local" exponential transform methods is that in the new methods, the computer code automatically calculates all of the biasing parameters in terms of the solution of the multigroup adjoint diffusion calculation; all of the guesswork is removed from the 
algorithm. Moreover, these parameters are "local" in the sense that they are defined differently in each spatial cell and in each energy group. Therefore, particles are biased not only according to space, but also angle and energy. This is in close accordance with the operation of the zerovariance method.

To describe the LET method in somewhat more detail, let us consider the transport problem

$$
\begin{aligned}
& \underline{\Omega} \cdot \underline{\nabla} \psi(\underline{r}, \underline{\Omega})+\sigma_{t} \psi(\underline{r}, \underline{\Omega})=\frac{\sigma_{s}}{4 \pi} \int \psi\left(\underline{r}, \underline{\Omega^{\prime}}\right) d \Omega^{\prime} \quad, \quad \underline{r} \in D,
\end{aligned}
$$

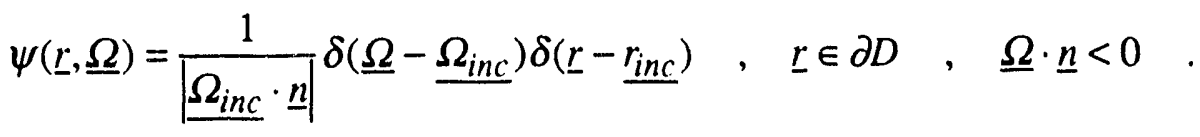

Here $D$ is the spatial domain, $\underline{r_{i n c}}$ is a point on the boundary $\partial D$, and $\underline{\Omega}_{\text {inc }}$ is a unit vector pointing inward at $\underline{r_{i n c}}$. This problem is driven by a unit-current pencil beam at $\underline{r_{\text {inc }}}$ in the incident direction $\Omega_{\text {inc }}$. Suppose that we wish to calculate

$$
J=\int_{\underline{r} \in R} \int_{\underline{\Omega} \cdot \underline{\underline{\Omega}}>0} \underline{\Omega} \cdot \underline{n} \psi(\underline{r}, \underline{\Omega}) d \Omega d r \quad,
$$

where $R$ is a portion of the outer boundary of the system. Thus, $J$ is the current exiting through $R$ due to an incident particle at $\underline{r_{\text {inc }}}$ travelling in the direction $\Omega_{\text {inc }}$.

To formulate a zero-variance method for this problem, let us define an adjoint transport problem

$$
\begin{gathered}
\underline{-\underline{\Omega}} \cdot \underline{\nabla} \psi^{*}(\underline{r}, \underline{\Omega})+\sigma_{t} \psi^{*}(\underline{r}, \underline{\Omega})=\frac{\sigma_{s}}{4 \pi} \int \psi^{*}\left(\underline{r}, \underline{\Omega^{\prime}}\right) d \Omega^{\prime}, \quad \underline{r} \in D, \\
\psi^{*}(\underline{r}, \underline{\Omega})= \begin{cases}1, & \underline{r} \in R, \underline{\Omega} \cdot \underline{n}>0, \\
0, & \underline{r} \in \partial D-R, \underline{\Omega} \cdot \underline{n}>0 .\end{cases}
\end{gathered}
$$

Then, if we define a function $\xi$ by

$$
\xi(\underline{r}, \underline{\Omega})=\psi(\underline{r}, \underline{\Omega}) \psi^{*}(\underline{r}, \underline{\Omega})
$$

one can show that $\xi$ satisfies the forward transport problem 


$$
\begin{gathered}
\underline{\Omega} \cdot \underline{\nabla} \xi(\underline{r}, \underline{\Omega})+\bar{\sigma}_{t}(\underline{r}, \underline{\Omega}) \xi(\underline{r}, \underline{\Omega})=\frac{1}{4 \pi} \int \bar{\sigma}_{s}\left(\underline{r}, \underline{\Omega^{\prime}}, \underline{\Omega}\right) \xi\left(\underline{r}, \underline{\Omega^{\prime}}\right) d \Omega^{\prime} \quad, \quad \underline{r} \in D, \\
\xi(\underline{r}, \underline{\Omega})=\frac{1}{\underline{\Omega_{i n c}} \cdot \underline{n}} \delta\left(\underline{\Omega}-\underline{\Omega_{i n c}}\right) \delta\left(\underline{r}-\underline{r_{i n c}}\right) \psi^{*}\left(\underline{r_{i n c}}, \underline{\Omega_{i n c}}\right), \underline{r} \in \partial D, \underline{\Omega} \cdot \underline{n}<0,
\end{gathered}
$$

with modified cross sections defined by

$$
\begin{gathered}
\bar{\sigma}_{t}(\underline{r}, \underline{\Omega})=\sigma_{t}-\underline{\Omega} \cdot \underline{\nabla} \ln \psi^{*}(\underline{r}, \underline{\Omega}), \\
\bar{\sigma}_{s}\left(\underline{r}, \underline{\Omega^{\prime}}, \underline{\Omega}\right)=\sigma_{s} \frac{\psi^{*}(\underline{r}, \underline{\Omega})}{\psi^{*}\left(\underline{r}, \underline{\Omega^{\prime}}\right)} .
\end{gathered}
$$

One can also show that analog Monte Carlo applied to the above problem for $\xi$ is a zero-variance method, in the following sense: every $\xi$-particle that enters the system has the same weight, and this weight never changes in a collision (the system is conservative); $\xi$-particles cannot leak out of the system except through $R$; and when they finally do leak out of the system (through $R$ ), their weight is identical to their original weight, $\psi^{*}\left(\underline{r_{i n c}}, \underline{\Omega_{i n c}}\right)$, which can be shown to be the exact value of the functional $J$. The difficulty with this method is that to implement it, one must know exactly the pointwise values of $\psi^{*}(\underline{r}, \underline{\Omega})$. (In addition, if one did know $\psi^{*}(\underline{r}, \underline{\Omega})$, one would immediately know $\psi^{*}\left(\underline{r_{i n c}}, \underline{\Omega_{i n c}}\right)$, and hence $J$. $)$

In the LET method [14,15], we approximate this impractical zero-variance method by replacing $\psi^{*}(\underline{r}, \underline{\Omega})$ with a suitable low-order approximation. We do this by the following procedure:

1. Replace problem (3) by its $P_{1}$ (or some other suitable low-order) approximation, and solve this problem numerically on a spatial grid. The solution of this problem will be, for example, the approximate (numerical) values of the adjoint scalar flux at the cell vertices.

2. Construct a continuous approximation to $\psi^{*}(\underline{r}, \underline{\Omega})$, valid within each spatial cell, using the numerical results derived in step 1 . In our work, this approximation varies exponentially in space, and it can either be isotropic in angle, or it takes the angular shape of a "Case"-discrete angular eigenfunction.

3. Within each cell, implement Eqs. (4)-(6), with $\psi^{*}(\underline{r}, \underline{\underline{s}})$ replaced by its approximation derived in step 2.

4. Use Monte Carlo with survival biasing to generate the solution of the modified Eqs. (5) for $\xi$. 
Through implementation and testing, we have shown that the Local Exponential Transform methods, both with and without angular biasing, are very efficient variance reduction techniques for the Monte Carlo simulation of multigroup one-dimensional and $x, y$-geometry transport problems. Numerical testing has repeatedly shown that the new methods have a significantly better figure of merit than conventional Splitting with Russian Roulette.

We shall present numerical results obtained using three variance reduction methods: two Local Exponential Transform methods (one with angular biasing, and one without), and Splitting with Russian Roulette. We have modified ANDY, a multigroup general geometry Monte Carlo Code, to include the Local Exponential Transform methods. ANDY was originally written at Los Alamos National Laboratory to solve multigroup general geometry problems with $S_{N}$ scattering cross section treatment. In the Splitting with Russian Roulette calculations, we used the weight window as it is implemented in MCNP [13], with the adjoint diffusion solution as the importance function for determining the splitting ratios. Also, in the LET calculations we used a local weight cutoff.

Figure 2: X,Y-Geometry Monte Carlo Problems 1-5

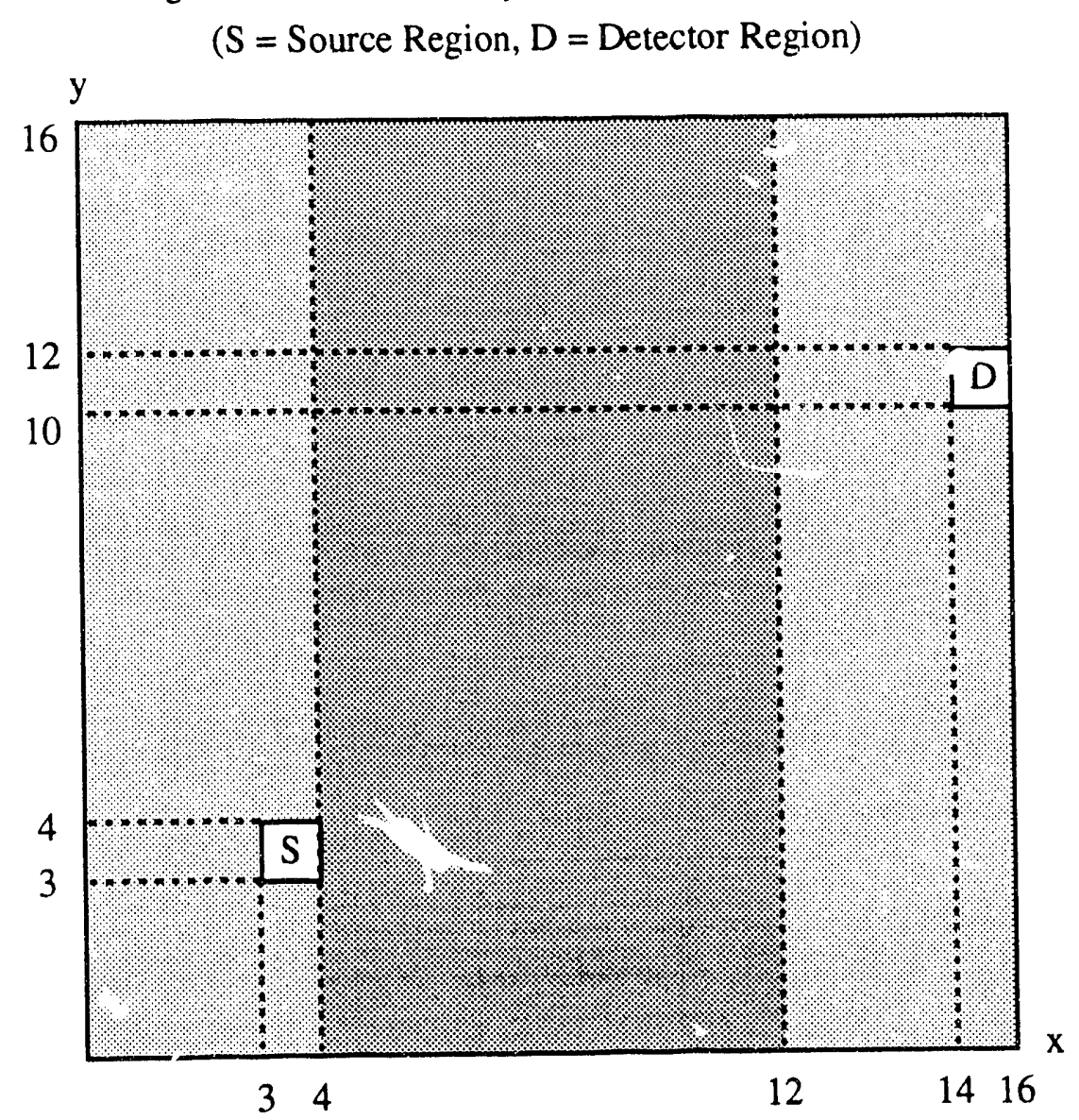


We consider five two-group $x, y$-geometry problems. For each of these five problems, $\sigma_{t, 1}=\sigma_{t, 2}=1 \mathrm{~cm}^{-1}$ and $\sigma_{s, 2 \rightarrow 1}=0$ (downscattering only), scattering is isotropic, the system is a square (see Figure 2) defined by $(0<x<16,0<y<16)$, a uniform source exists for group 1 in the square subregion $(3<x<4,3<y<4)$, and we wish to compute the group 2 detector response for the square subregion $(14<x<16,10<y<12)$. Problems 1 through 4 are for a homogeneous $16 \mathrm{~cm} \times 16 \mathrm{~cm}$ rectangle, while problem 5 is for a heterogeneous rectangle consisting of the three subregions $(0<x<4,0<y<16),(4<x<12,0<y<16)$, and $(12<x<16,0<y<16)$. The cross sections for these problems are given in Table 1. To calculate the adjoint diffusion solutions for these problems, we divided the system into $8 \times 8$ cells with $\Delta x=\Delta y=2 \mathrm{~cm}$.

Table 1: Configuration for Monte Carlo Problems 1-5 $\left(\sigma\right.$ in $\left.\mathrm{cm}^{-1}\right)$

\begin{tabular}{|c|c|c|c|c|c|c|c|c|c|}
\hline & Region $(\mathrm{cm})$ & $\sigma_{s, 1 \rightarrow 1}$ & $\sigma_{s, 1 \rightarrow 2}$ & $\sigma_{a, 1}$ & $\sigma_{t, 1}$ & $\sigma_{s, 2 \rightarrow 2}$ & $\sigma_{s, 2 \rightarrow 1}$ & $\sigma_{a, 2}$ & $\sigma_{t, 2}$ \\
\hline 1 & $0<x<16,0<y<16$ & .90 & .08 & .02 & 1.00 & .90 & 0.00 & .10 & 1.00 \\
\hline 2 & $0<x<16,0<y<16$ & .10 & .10 & .80 & 1.00 & .10 & 0.00 & .90 & 1.00 \\
\hline 3 & $0<x<16,0<y<16$ & .90 & .08 & .02 & 1.00 & .10 & 0.00 & .90 & 1.00 \\
\hline 4 & $0<x<16,0<y<16$ & .10 & .10 & .80 & 1.00 & .90 & 0.00 & .10 & 1.00 \\
\hline \multirow{3}{*}{5} & $0<x<4,0<y<16$ & .90 & .08 & .02 & 1.00 & .90 & 0.00 & .10 & 1.00 \\
\hline & $4<x<12,0<y<16$ & .10 & .10 & .80 & 1.00 & .10 & 0.00 & .90 & 1.00 \\
\hline & $12<x<16,0<y<16$ & .90 & .08 & .02 & 1.00 & .90 & 0.00 & .10 & 1.00 \\
\hline
\end{tabular}

For Problems 1-5 we list in Table 2 the Benefit factors $B$ (defined as the conventional figure-of-merit divided by the figure-of-merit for Monte Carlo with survival biasing only) for the LET methods and Splitting with Russian Roulette. In each of the above problems, the LET method with angular biasing outperforms the LET method without angular biasing (particularly for highly scattering problems), which in turn outperforms Splitting with Russian Roulette. We also note that the Local Exponential Transform methods require significantly less static computer storage than Splitting with Russian Roulette because they do not require the storage of any daughter particles that would arise from splitting. 
Table 2: Benefit Factors for Monte Carlo Problems 1-5

\begin{tabular}{|c|c|c|c|c|}
\hline & \multirow{2}{*}{$\begin{array}{l}\text { Problem } \\
\text { Description }\end{array}$} & \multicolumn{2}{|c|}{ Local Exopnential Transform } & \multirow{2}{*}{$\begin{array}{l}\text { Splitting With } \\
\text { Russian Roulette }\end{array}$} \\
\hline & & No Angular Bias & With Angular Bias & \\
\hline 1 & $c_{1}=c_{2}=0.9$ & 6.4 & 79.0 & 3.7 \\
\hline 2 & $c_{1}=c_{2}=0.1$ & 1160.3 & 5286.2 & 490.5 \\
\hline 3 & $c_{1}=0.9, c_{2}=0.1$ & 45.7 & 135.7 & 7.9 \\
\hline 4 & $c_{1}=0.1, c_{2}=0.9$ & 12.2 & 193.6 & 3.5 \\
\hline 5 & heterogeneous & 131.6 & 410.2 & 25.0 \\
\hline
\end{tabular}

2C. Deterministic $S_{\mathrm{N}}$ : Acc ieration of Advanced Differencing Schemes for the Discrete Ordinates Equations

Diffusion Synthetic Acceleration (DSA) has become a very useful tool for accelerating the iterative convergence of discrete ordinates problems [16-18]. However, the method has some limitations that have prevented its wide application in the most difficult problems, namely multidimensional problems using new, sophisticated, and highly-accurate spatial differencing schemes. These difficulties exist even for rectangular spatial grids; the added complication of a nonrectangular grid makes matters even worse. In some of our recent work, we have overcome some of these difficulties and have developed efficient ways of implementing DSA in multidimensional geometries for highly accurate differencing schemes.

To describe our results, we must first convey the basic ideas behind DSA. Thus, let us suppose that we wish to solve the transport equation

$$
\mu \frac{\partial \psi}{\partial x}(x, \mu)+\sigma_{t}(x) \psi(x, \mu)=\frac{\sigma_{s}(x)}{2} \int_{-1}^{1} \psi(x, \mu) d \mu+Q(x)
$$

with unspecified boundary conditions. The standard Source Iteration (SI) scheme is defined by

$$
\begin{gathered}
\mu \frac{\partial \psi^{(\ell+1 / 2)}}{\partial x}(x, \mu)+\sigma_{l}(x) \psi^{(\ell+1 / 2)}(x, \mu)=\frac{1}{2}\left[\sigma_{s}(x) \phi_{0}^{(\ell)}(x)+Q(x)\right] \\
\phi_{0}^{(\ell+1 / 2)}(x) \equiv \int_{-1}^{1} \psi^{(\ell+1 / 2)}(x, \mu) d \mu
\end{gathered}
$$




$$
\phi_{0}^{(\ell+1)}(x)=\phi_{0}^{(\ell+1 / 2)}(x)
$$

Here $\ell$ denotes the iteration index. We wish to replace Eq. $(8 c)$ by another equation which may be more complex, but which nevertheless leads to a much more rapidly converging algorithm. If we subtract Eq. (8a) from Eq. (7) and define the residual $f$ as

$$
f(x, \mu)=\psi(x, \mu)-\psi^{(\ell+1 / 2)}(x, \mu)
$$

then we obtain the following exact equation for $f$ :

$$
\mu \frac{\partial f}{\partial x}\left(y_{r}, \mu\right)+\sigma_{t}(x) f(x, \mu)-\frac{\sigma_{S}(x)}{2} \int_{-1}^{1} f\left(x, \mu^{\prime}\right) d \mu^{\prime}=\frac{\sigma_{S}(x)}{2}\left[\phi_{0}^{(\ell+1 / 2)}(x)-\phi_{0}^{(\ell)}(x)\right]
$$

If we solve Eq. (10) and then use Eq. (9), we obtain the converged solution of the problem. Of course, this is not feasible because Eq. (10) for $f$ is just as difficult to solve as the original transport equation (7) for $\psi$. The idea of DSA is to replace the transport operator on the left side of Eq. (10) by its diffusion description. Since the resulting $f$ is approximate, this procedure will give an approximate, rather than an exact $\psi$. Thus, Eqs. (10) and (9) yield:

$$
\begin{gathered}
-\frac{d}{d x} \frac{1}{3 \sigma_{l}(x)} \frac{d}{d x} f_{0}^{(\ell+1)}(x)+\sigma_{a}()^{*} f_{0}^{(\ell+1)}(x)=\sigma_{s}(x)\left[\phi^{(\ell+1 / 2)}(x)-\phi^{(\ell)}(x)\right], \\
\phi^{(\ell+1)}(x)=\phi^{(\ell+1 / 2)}(x)+f_{0}^{(\ell+1)}(x) .
\end{gathered}
$$

Eqs. $(8 a, b)$ and $(11 a, b)$ are the DSA equations. For sufficiently fine spatial grids, these (discretized) equations produce a very rapidly converging iterative algorithm. However, for a diffusion differencing scheme chosen arbitrarily, the method usually diverges for spatial grids larger than about one mean free path thick. The main difficulty is: for a given transport differencing scheme for Eqs. $(8 a, b)$, can one determine a discretized version of Eqs. $(11 a, b)$ in which the resulting discretized DSA algorithm is stable and efficient for all mesh sizes?

We have been engaged in a research program for severai years involving the development of new transport differencing schemes and ways of asymptotically analyzing how these schemes behave in terms of accuracy for optically thick spatial cells [19-22]. We and other workers have used these asymptotic methods to develop new classes of differencing schemes that are reasonably simple, yet much more accurate than previous methods. We have also recently shown how these schemes can be efrectively acceierated using a modifieu fómin of DSA. The basic concept is that 
one starts with the original discretized transport equation $(8 a, b)$. Then, one formulates the exact discretized equation for the residual [Eq. (10)]. Then one takes the $\mathrm{P}_{1}$ approximation of this discretized problem. This yields a system of equations of the form

$$
\begin{gathered}
\frac{d}{d x} f_{1}^{(\ell+1)}(x)+\sigma_{a}(x) f_{0}^{(\ell+1)}(x)=\sigma_{s}(x)\left[\phi^{(\ell+1 / 2)}(x)-\phi^{(\ell)}(x)\right], \\
\frac{1}{3} \frac{d}{d x} f_{0}^{(\ell+1)}(x)+\sigma_{t}(x) f_{1}^{(\ell+1)}(x)=0, \\
\phi^{(\ell+1)}(x)=\phi^{(\ell+1 / 2)}(x)+f^{(\ell+1)}(x),
\end{gathered}
$$

which generally cannot be algebraically manipulated into the form of a discretized diffusion equation with scalar flux unknowns. However, these equations are definitely a discretized version of the $P_{1}$ equations. The resulting discretized $P_{1}$ problem is, for the sche. nes we have considered, self-adjoint. Therefore, one can use the conjugate gradient method to solve these equations, provided the equations are also positive-definite. Although we cannot prove that they are positivedefinite, the resulting CG method works as efficiently as one would expect if the equicions were known to be positive-definite. In fact, the efficiency of the conjugate-gradiant scheme for these equations is remarkable, given the historical emphasis that has previously been placed on obtaining a discretized DSA diffusion problem that can be solved efficiently with multigrid techniques [6].

2D. Deterministic $\mathrm{S}_{\mathrm{N}}$ : Quasidiffusion Transport Methods

The Quasidiffusion (QD) method, originally proposed by Gol'din [23], is a nonlinear iterative scheme for solving uransport problems. Like Diffusion Synthetic Acceleration (DSA), each QD iteration consists of one transport sweep foliowed by one diffusion calculation. In theory and in practice, the QD method exhibits about the same convergence rate as DSA [24]. Unlike DSA, the QD method does not require the transport and diffusion calculations to be discretized consistently, and the multidimensional QD method is more easily implemented, particularly on nonrectangular grids. However, the multidimnsional QD diffusion problem is non-selfadjoint; this causes difficulties in the discretization and iteration procedures for solving these problems. For example, conjugate gradient and multigrid methods do not always perform efficiently for such problems.

To overcome this difficulty, we have developed and implemented a "symmetrized" Quasidiffusion (SQD) method in which the non-selfadjoint QD diffusion problem is replaced by an 
equivalent system of two selfadjoint diffusion problems. These problems are lower-triangular in form; the solution of the first problem is required for solving the second problem, but not vice versa. For multidimensional geometries, these problems are more easily discretized (using a variational method) and more efficiently solved (using the standard conjugate-gradient method) than in the standard QD method. We have implemented the SQD method and have obtained calculational results for transport problems in: $x, y$ - geometry $[25,26]$.

To describe our results, we must begin by developing the QD method. For simplicity, let us consider a one-group general-geometry transport problem with isotropic scattering and interior source $Q(\underline{x})$, and a prescribed incident flux $\psi^{b}(\underline{x}, \underline{\Omega})$ at the outer boundary:

$$
\begin{gathered}
\Omega_{i} \frac{\partial \psi}{\partial x_{i}}(\underline{x}, \underline{\Omega})+\sigma_{t}(\underline{x}) \psi(\underline{x}, \underline{\Omega})=\frac{\sigma_{s}(\underline{x})}{4 \pi} \phi(\underline{x})+\frac{Q(\underline{x})}{4 \pi} \quad, \quad \underline{x} \in D, \\
\psi(\underline{x}, \underline{\Omega})=\psi^{b}(\underline{x}, \underline{\Omega}) \quad, \quad \underline{x} \in \partial D \quad, \quad \underline{\Omega} \cdot \underline{n}<0 .
\end{gathered}
$$

Here we have used the summation convention: repeated subscripts are summed from 1 to 3 . Also, $\phi=\int \psi d \Omega$ is the scalar flux. Operating on Eq. $(13 a)$ by $\int(\cdot) d \Omega$ and $\int \underline{\Omega}(\cdot) d \Omega$, we obtain

$$
\begin{aligned}
\frac{\partial J_{i}}{\partial x_{i}}+\sigma_{a} \phi & =Q, \\
\frac{\partial}{\partial x_{i}} \int \Omega_{j} \Omega_{i} \psi d \Omega+\sigma_{t} J_{j} & =0 \quad, \quad 1 \leq j \leq 3
\end{aligned},
$$

where $J_{j}=\int \Omega_{j} \psi d \Omega$ is the $j$-th component of the current. Thus, if we define the symmetric positive-definite "Eddington" tensor

$$
E_{i j}(\underline{x})=\frac{\int \Omega_{i} \Omega_{j} \psi(\underline{x}, \underline{\Omega}) d \Omega}{\int \psi(\underline{x}, \underline{\Omega}) d \Omega}
$$

and eliminate the current terms between Eqs. (14) and (15), we obtain

$$
-\frac{\partial}{\partial x_{i}} \frac{1}{\sigma_{t}(\underline{x})} \frac{\partial}{\partial x_{j}} E_{i j}(\underline{x}) \phi(\underline{x})+\sigma_{a}(\underline{x}) \phi(\underline{x})=Q(\underline{x})
$$

Given $\underline{E}$, Eq. (17) - with suitable boundary conditions - determines $\phi$. To obtain such boundary conditions, we multiply Eq. (13b) by $2\left|\Omega_{i} n_{i}\right|$ and integrate over the incoming directions: 


$$
2 \underset{\Omega_{i} n_{i}<0}{\int}\left|\Omega_{i} n_{i}\right| \psi^{b}(\underline{x}, \underline{\Omega}) d \Omega=\int_{4 \pi}\left(\left|\Omega_{i} n_{i}\right|-\Omega_{i} n_{i}\right) \psi d \Omega=\frac{\int \Omega_{i} n_{i} \mid \psi d \Omega}{\int_{4 \pi} \psi d \Omega} \phi-n_{i} J_{i}
$$

Hence, if we define the "boundary Eddington factor"

$$
B(\underline{x})=\frac{\iint_{4 \pi} \Omega_{i} n_{i} \mid \psi d \Omega}{\int_{4 \pi} \psi d \Omega}, \quad \underline{x} \in \partial D
$$

and we use Eq. (15) to eliminate $J$ from Eq. (18), we obtain

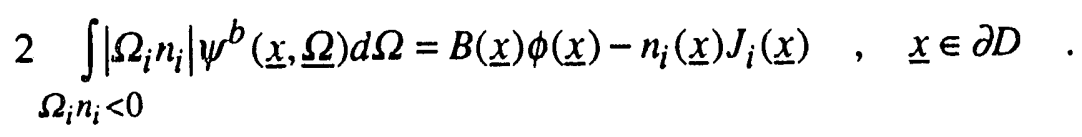

The QD metriod is defined as follows: (i) Given an estimate $\phi$ of the scalar flux, one solves Eqs. (13) to obtain a new estimate for $\psi$. (ii) This result is used in Eqs. (16) and (19) to calculate new estimates for $E_{i j}$ and $B$. (iii) These results are then used in Eqs. (17) and (20) to calculate a new estimate for $\phi$. This process repeats until convergence. [Note: the boundary condition (20) agrees with the previous formulation given by Gol'din only for a vacuum boundary. For nonvacuum boundaries, Eq. (20) is a more accurate and efficient (in terms of iteration performance) boundary condition.]

In practice, the QD method converges rapidly because the functionals $\underline{E}$ and $B$ depend weakly on $\psi$. In fact, if $\psi$ is a linear function of angle, $\underline{E}$ and $B$ are both independent of $\psi$, $E_{i j}=\delta_{i j} / 3$ and $B=1 / 2$, and the diffusion problem (17), (20) exactly reduces to the familiar diffusion equation with Marshak boundary conditions.

However, in multidimensional geometries, the diffusion problem (17), (20) for $\phi$ is nonselfadjoint. This creates difficulties for the discretization and solution procedures. Hence, we have developed a "symmetrized" Quasidiffusion method, which replaces the non-selfadjoint problem by two selfadjoint problems. For any function $\xi(\underline{x})$, we have

$$
\frac{\partial}{\partial x_{j}} E_{i j} \phi=\frac{\partial}{\partial x_{j}}\left(E_{i j} e^{-\xi}\right)\left(e^{\xi} \phi\right)=\left(\frac{\partial E_{i j}}{\partial x_{j}}-E_{i j} \frac{\partial \xi}{\partial x_{j}}\right) \phi+\left(E_{i j} e^{-\xi}\right) \frac{\partial}{\partial x_{j}}\left(e^{\xi} \phi\right) .
$$


Hence, if we require $\xi(\underline{x})$ to satisfy the following (selfadjoint) diffusion problem

$$
\begin{gathered}
\frac{\partial}{\partial x_{i}} \frac{\phi}{\sigma_{l}}\left(\frac{\partial E_{i j}}{\partial x_{j}}-E_{i j} \frac{\partial \xi}{\partial x_{j}}\right)=0, \quad \underline{x} \in D, \\
n_{i}\left(\frac{\partial E_{i j}}{\partial x_{j}}-E_{i j} \frac{\partial \xi}{\partial x_{j}}\right)=0, \quad \underline{x} \in \partial D,
\end{gathered}
$$

then Eqs. (17) and (20) reduce to the following (selfadjoint) diffusion problem for $e^{\xi} \phi$ :

$$
\begin{gathered}
-\frac{\partial}{\partial x_{i}}\left(\frac{E_{i j} e^{-\xi}}{\sigma_{t}}\right) \frac{\partial}{\partial x_{j}}\left(e^{\xi} \phi\right)+\left(\sigma_{a} e^{-\xi}\right)\left(e^{\xi} \phi\right)=Q \quad, \quad \underline{x} \in D, \\
2 \underset{\substack{\Omega_{i} n_{i}<0}\left|\Omega_{i} n_{i}\right| y^{b} d \Omega 2}{ }=\left(B e^{-\xi}\right)\left(e^{\xi} \phi\right)+\frac{e^{-\xi}}{\sigma_{t}} n_{i} E_{i j} \frac{\partial}{\partial x_{j}}\left(e^{\xi} \phi\right), \quad \underline{x} \in \partial D
\end{gathered}
$$

The SQD method is now defines sollows. (i) Given an estimate $\phi$ of the scalar flux, one solves Eqs. (13) to obtain a new estimate for $\psi$. (ii) This result is used in Eqs. (16) and (19) to calculate new estimates for $\underline{\underline{E}}$ and $B$, and to construct a new scalar flux estimate $\phi$. (iii) These results are used in Eqs. (22), which are solved for $\xi$. (iv) These results are then used in Eqs. (23), which are solved for $e^{\xi} \phi$. One then trivially obtains a new estimate for $\phi$, and the process repeats until convergence.

We have implemented the SQD method in an $x, y$-geometry rectangular-mesh test code, using the short characteristic method (with unknowns at cell vertices) for the transport sweeps, and cell-vertex finite-difference schemes for Eqs. (22) for $\xi$, and (23) for $\phi$. The diffusion schemes were obtained using a variational principle, which exists because the diffusion problems for $\xi$ and $\phi$ are selfadjoint. The resulting discretized diffusion problems are selfadjoint and were efficiently solved using the conjugate gradient method.

For fine spatial meshes, the numerical results that we have obtained have nearly the same accuracy as that of the diamond difference scheme, and the number of transport iterations required for convergence is also nearly identical. However, as the spatial meshes become coarse, the Diamond solutions begin to degrade. In addition, the diamond-differenced code TWODANT [27] uses a negative flux fixup, and this fixup interferes with both the accuracy and the iterative convergence of the code. Thus, as the spatial meshes become thicker, the performance of TWODANT, with regard to accuracy and speed of convergence, begins to seriously degrade. 
However, the SQD method converges equally rapidly for all mesh sizes, and the solution remains monotonic and accurate for much coarser meshes than that of the diamond scheme.

For example, the number of transport iterations and the absorption rate in a subregion of a typical $x, v$-geometry problem with a series of uniform rectangular spatial grids are given in Table 3. The problem consists of a homogeneous square region $(0<x<8,0<y<8)$ having $\sigma_{t}=1.0$, $\sigma_{s}=1.0, Q=1.0$ for $(0<x<2,0<y<2)$, and $Q=0$ outside this inner region. The dimensions of : and $y$ are $\mathrm{cm}$; the dimensions of $\sigma$ are $\mathrm{cm}^{-1}$. The top and right boundaries of the system are vacuum; the bottom and left are reflecting. We used the standard $\mathrm{S}_{8}$ Gauss-Legendre Quadrature set, with a relative pointwise convergence criterion of $10^{-5}$.

Table 3: SQD Resılts

\begin{tabular}{|l|c|c|c|c|}
\hline & \multicolumn{2}{|c|}{ Transport Iterations } & \multicolumn{2}{|c|}{ Relative Error in $\int_{66}^{8} \int_{a}^{8} \sigma_{a} \phi d x d y$} \\
\hline $\begin{array}{l}\Delta x=\Delta y \\
\text { (in mfp) }\end{array}$ & DD-DSA & SQD & DD-DSA & SQD \\
\hline 0.125 & 8 & 7 & $-3.61 \mathrm{E}-4$ & $-3.61 \mathrm{E}-4$ \\
0.25 & 8 & 8 & $-1.71 \mathrm{E}-3$ & $-1.17 \mathrm{E}-3$ \\
0.5 & 8 & 8 & $-7.31 \mathrm{E}-3$ & $-2.17 \mathrm{E}-3$ \\
1.0 & 9 & 8 & $-3.96 \mathrm{E}-2$ & $-3.70 \mathrm{E}-3$ \\
2.0 & 12 & 8 & $-1.97 \mathrm{E}-1$ & $-2.10 \mathrm{E}-2$ \\
\hline
\end{tabular}

We see that the relative errors in the DD-DSA and SQD solutions are the same for the finest mesh, and that both methods require nearly the same number of iterations to converge. However, the DD-DSA method requires more iterations for larger spatial meshes because of the negative flux fixups, and the accuracy of this scheme degrades faster for coarser meshes. We have also observed that the SQD pointwise solutions are monotonic (they exhibit no spurious highfrequency oscillations) and overall tend to be more accurate than the DD solution for coarser meshes. 


\section{E. Monte Carlo: Response History Method for Electron Transport}

The original UM proposal was written in January 1991 and since that time there has been substantial progress on this task which is discussed below. However, due to the absence of DOE funding for this task, this effort has been partially supported by other sources, and we have asked that the funds for this task be restored for the next year of the contract, in order to complete this task. Although this work was not supported by the current grant, progress on it and related work will be included in this section since we are asking that it be included in the next year's project. The progress will be divided into three sections: (1) the original response history work that was completed in August 1991, (2) the implementation of the response history metho $\dot{j}$ into the production Monte Carlo code MCNP during the summer 1992, and (3) the utilization of massively parallel processors for Monte Carlo that has been the subject of recent effort.

\section{Original Response History Effort}

The doctoral work of C.T. Ballinger [28], who obtained his Ph.D. in August 1991, demonstrated the promise of the response history Monte Carlo (RHMC) method (this method was previously called the "response matrix" method) for simulation of electron transport problems. In this technique [28-29], a pre-run-time, highly accurate (but time consuming), single scattering Monte Carlo calculation is performed, tracking electrons passing through hemispherical blocks of material. The state variables (position, energy, and direction) of electrons of various initi i' energies are tabulated as they exit hemispheres of a preset radius. It should be soteu that the single scatter code needs to be used only once for each different material, and these "response pdfs" can be stored in a library to be used in subsequent analyses, perhaps years later on completely different configurations. This is similar to the way condensed history kernels are used over and over again, the difference being that the response history distribution functions are generated by a single scatter Monte Carlo code, hence are not limited by any restrictive assumptions to make the theory valid. Figure 3 illustrates this single scatter, or local, Monte Carlo calculation. 
Figure 3. Response History PDFs

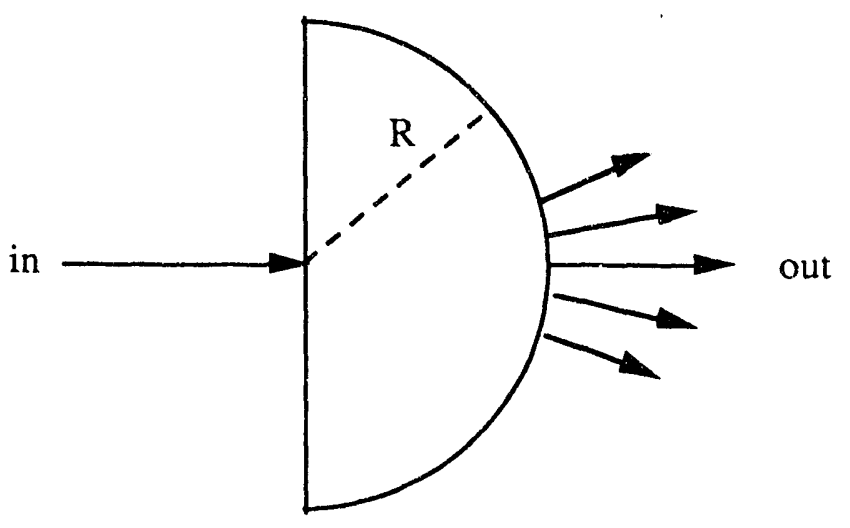

At run time, denoted the global calculation, electrons are tracked by modeling them as moving through hemispheres one at a time, sampling for the new state variables as they exit each hemisphere. Since the distribution functions describing the state variables were generated stochastically by tallying actual tracks, the straight path assumption which limits the condensed history methodology is not a concern. A particle proceeds through a macroscopic volume by stacking the hemispheres dynamically, with each new hemisphere oriented in the direction along which the electron exited the previous surface. Figure 4 illustrates the concept for combining hemispheres for the global calculation.

Figure 4. Recursive Use of Response History PDFs

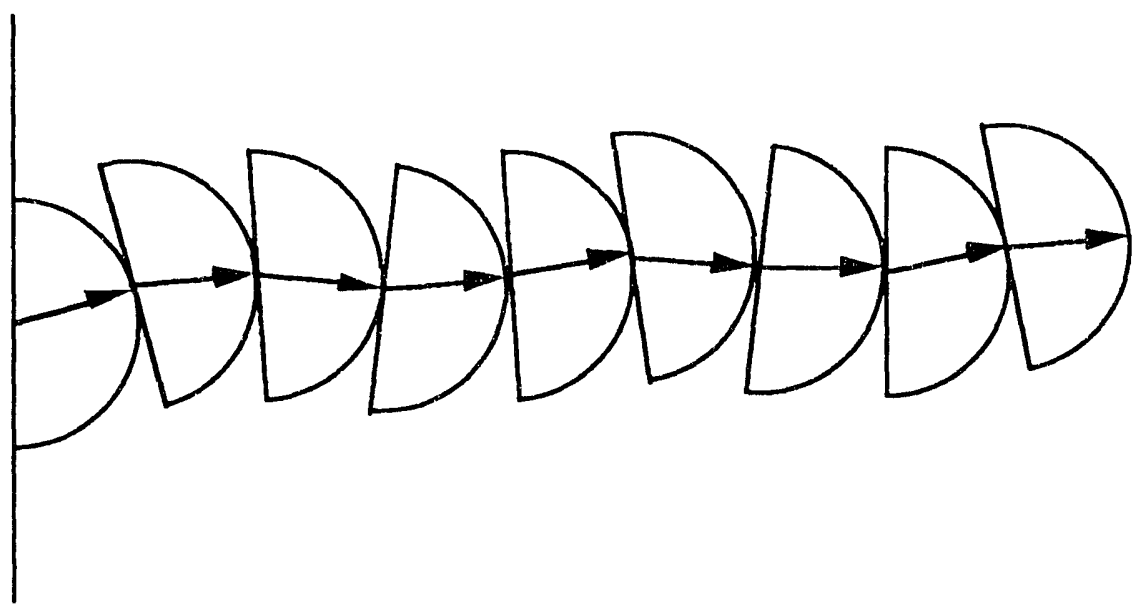

The doctoral studies of Ballinger [28] demonstrated that a high degree of accuracy can be achieved using this technique, especially at low electron energies, in direct contrast to condensed history $(\mathrm{CH})$ based Monte Carlo codes. To illustrate this, Figure 5 compares the RHMC method 
versus (1) experimental data for $50 \mathrm{keV}$ electrons incident on a thick target of aluminum, (2) analog (single scatter) Monte Carlo, and (3) $\mathrm{CH}$ methods as implemented in MCNP and SANDYL, which are production Monte Carlo codes with electron transport capability. Figure 6 is a similar comparison for gold. In both cases it is evident that the RHMC method gives more accurate results than the $\mathrm{CH}$ method, and compares well with both experimental data and analog Monte Carlo, for both aluminum and gold.

Figure 5. RHMC Backscatt

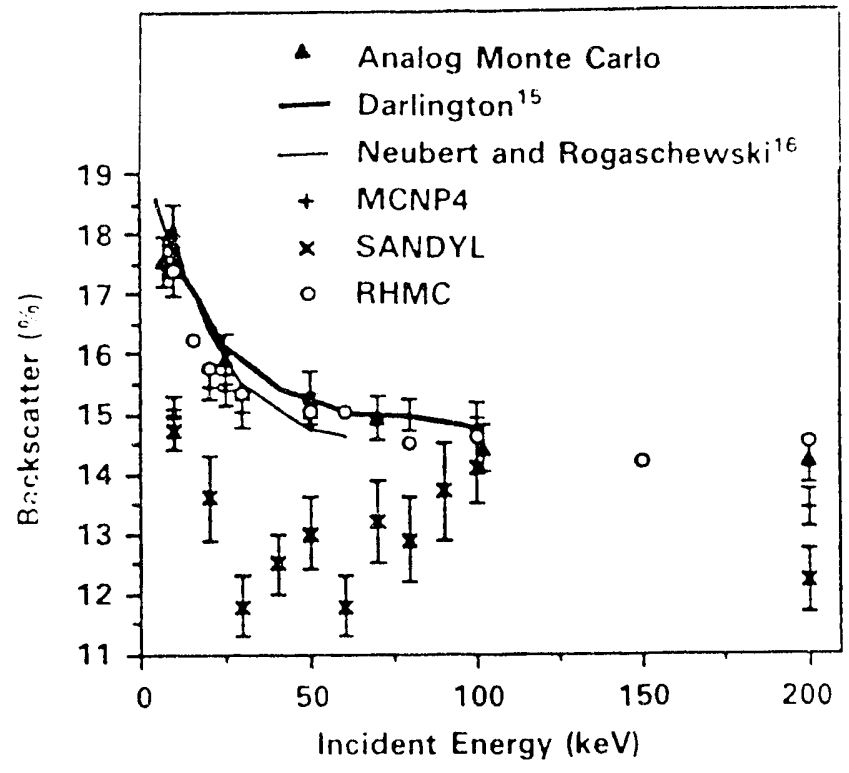

Figure 6. RHMC Backscatter Percentages for Electrons Incident on Gold

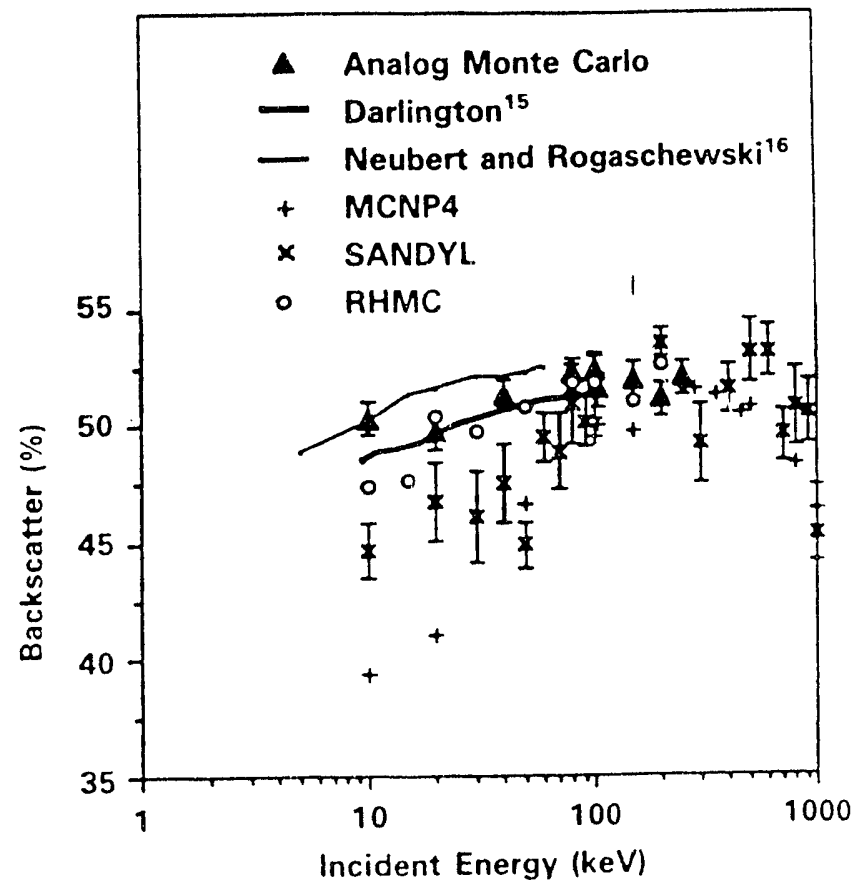


Finally, Table 4 gives some representative CPU times for the RHMC method vs. the CH method, and it can be seen that the RHMC method is consistently more efficient than the less accurate $\mathrm{CH}$ method, and is orders of magnitude faster than analog Monte Carlo, which it approaches in accuracy. The results in Figures 5 and 6 and Table 4 are taken from [29].

Table 4. Comparison of CPU Times for Backscatter Calculations

\begin{tabular}{|c|c|c|c|c|}
\hline $\begin{array}{c}\text { Energy } \\
\text { (keV) }\end{array}$ & Material & $\begin{array}{c}\text { MCNP4 } \\
\text { (CPU s) }\end{array}$ & $\begin{array}{c}\text { RHMC } \\
\text { (CPU s) }\end{array}$ & $\begin{array}{c}\text { Analog } \\
\text { Monte Carlo } \\
\text { (CPU s) }\end{array}$ \\
\hline 200 & Aluminum & 94.3 & 66.1 & 3400 \\
100 & Aluminum & 80.1 & 46.2 & 3260 \\
70 & Aluminum & 76.9 & 40.0 & 2800 \\
25 & Aluminum & 54.6 & 20.4 & 1490 \\
10 & Aluminum & 28.0 & 14.7 & 408 \\
250 & Gold & 110.9 & 63.5 & 18629 \\
200 & Gold & 101.0 & 62.9 & 15076 \\
100 & Gold & 85.5 & 58.1 & 11117 \\
80 & Gold & 78.9 & 54.4 & 578. \\
20 & Gold & 56.5 & 45.0 & 1441 \\
\hline
\end{tabular}

"Does not include CPU time required to perform local calculations.

This initial work on the RHMC method was essentially complete before the current reporting period started in January 1992, although these results were not available at the time that the proposal for this work was written in January 1991. Let us now discuss some of the progress that has been made since the original work by Ballinger.

\section{Implementation of Response History Method into MCNP}

The promise of the RHMC method for electron transport was sufficient to try to implement some of its features into MCNP. In effort carried out by Scott Turner, a UM graduate student in nuclear engineering, at LLNL during the summer of 1992, a version of MCNP with the RHMC method was developed and tested. In addition, to help address a recurring problem with the energy loss of electrons using the $\mathrm{CH}$ method (which is inherently energy-independent), the response pdf for energy loss (which arises naturally from the RHMC method) was implemented into MCNP and the preliminary results appear to be good. Thus the RHMC method has the potential of improving the overall electron transport simulation or just the energy loss portion of it with the $\mathrm{CH}$ method. 


\section{Utilization of MPPs for Monte Carlo}

Even with the development of the RHMC method, electron transport is still one of the most computationally-intensive applications in computational science. This has spurred investigation into the use of advanced computers for these intensive simulations, in particular massively parallel processors. It has been found over the past decade that Monte Carlo methods can be successfully adapted to vector architectures (e.g., Cray), but with an accompanying investment in effort that may be excessive.[31-32] On the other hand, Monte Carlo particle transport is an inherently parallel process, because individual particle "histories" or simulations, do not interact and one can perform many particle simulations simultaneously on different processors, making Monte Carlo an "inherently parallel" computational method for particle transport. (These remarks have to be tempered for criticality, feedback, and time-dependence, but in generai these effects do not seriously impact the basic independence of individual particle histories in a Monte Carlo simulation.) The basic conclusion is that particle transport Monte Carlo is readily suited for parallel architectures, and adaptable, but with considerable effort, to vector architectures.

The UM has made some recent computer acquisitions that have prompted our decision to look at these issues in some detail. The UM acquired a 32 node MPP in June 1992, a Kendall Square Research KSR-1, and earlier obtained an 8 node cluster of high performance IBM RS6000 workstations. The KSR-1 acquisition was part of a larger NSF grant that will eventually bring to the UM a parallel computing facility with the following parallel computers: (1) the KSR1, (2) a second MPP characterized by a distributed memory architecture, and (3) a large cluster of networked workstations that will be a considerable enhancement of the current 8 node cluster. Thus the UM is constructing a parallel computing facility that will allow access to a variety of parallel architectures.

Ongoing work in collaboration with Lawrence Livermore National Laboratory (LLNL) provided additional incentive for our effort in this area. A time-dependent photon transport Monte Carlo code (TPHOT) was parallelized for an arbitrary number of processors and implemented on the 128-processor BBN-AC1 TC2000 ("Butterfly") at LLNL and a large number of timing runs were made, varying both the number of processors (from 1 to 116) and the size of the simulation (i.e., the number of histories) over 5 orders of magnitude. This effort was carried out by J. Rathkopf at LLNL and a high school teacher, M. Litvin of Phillips Academy at Andover, Massachusetts. To complement this effort on the BBN, a version of TPHOT was implemented on the KSR-1 and a number of timing runs were performed for comparison to the BBN. (The KSR-1 implementation was done by Amit Majumdar, a graduate student in the Department of Nuclear Engineering.) 
The results of the BBN and KSR runs are plotted in Figures 7 and 8 respectively, which are plots of the speedups versus the number of processors for a wide range of workloads ( 1 to 100,000 in arbitrary units). We have also developed simple timing models for both the BBN and the KSR, and the computed speedups from these timing models seems to predict the obsi ved speedups quite nicely. These figures are taken from the paper that has been accepted for presentation at the Joint ANS-ENS International Conference on Mathematical Methods and Supercomputing in Nuclear Applications, to be held in Karlsruhe in April 1993, which summarizes the simple timing model and describes the parallel Monte Carlo algorithm that was used for these simulations. [33]

Figure 7. Measured and Predicted Speedups for BBN

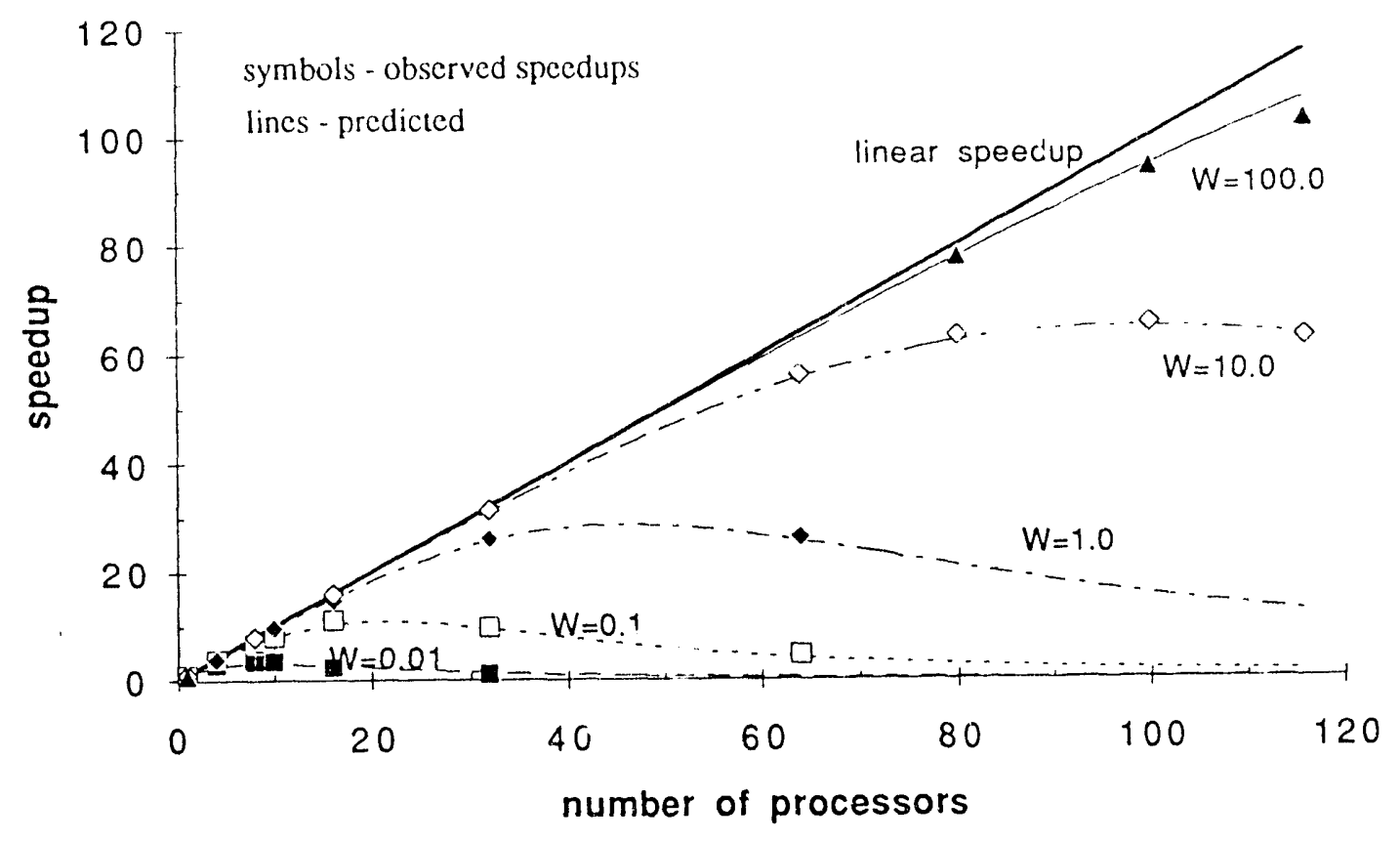


Figure 8. Measured and Predicted Speedups for KSR

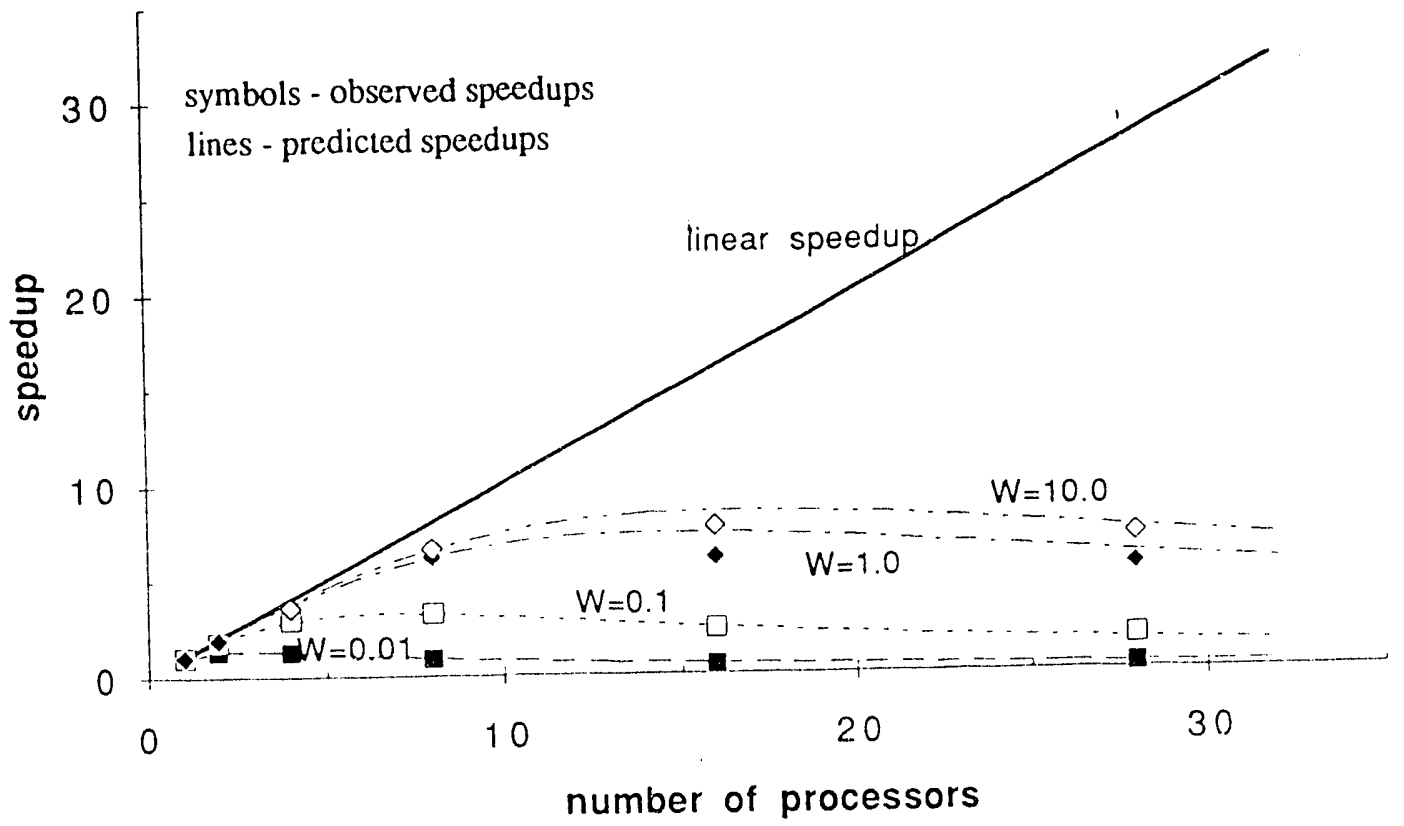




\section{Bibliography}

1. Dorning, J.J., "Modern Coarse-Mesh Methods - A Development of the 70's," Proc. Conf. Computational Methods in Nuclear Engineering 3, 1, Williamsburg VA, April 23-25, 1979 , American Nuclear Society (1979).

2. Lawrence, R.D., "Progress in Nodal Methods for the Solution of the Neutron Diffusion and Transport Equations," Prog. Nucl. Energy 17, 3, 271 (1986).

3. Altiparmakov, D.V. and Tomasevic, $\mathrm{Dj}$., "Variational Formulation ố a Higher Order Nodal Diffusion Method," Nucl. Sci. Eng. 105, 256 (1990).

4. McCormick, S., ed., Multigrid Methods, Society for Industrial and Applied Mathematics, Philadelphia (1987)

5. Briggs, W.L., A Multigrid Tutorial, Society for Industrial and Applied Mathematics, Philadelphia (1987).

6. Alcouffe, R.E., Brandt, A., J.E. Dendy, and J.W. Painter, "The Multi-Grid Method for the Diffusion Equation with Strongly Discontinuous Coefficients," SIAM J. Sci. Stat. Comput. 2, $430(1981)$.

7. R.P. Rulko and E.W. Larsen, "Variational Derivation and Numerical Analysis of $\mathrm{P}_{2}$ Theory in Planar Geometry," Nucl. Sci. Eng., to appear.

8. E.W. Larsen, J.M. McGhee, and J.E. Morel, "The Simplified $P_{N}$ Equations as an Asymptotic Limit of the Transport Equation," Trans. Am. Nucl. Soc. 66, 231 (1992). (This is an abridged version of Ref. 9.)

9. E.W. Larsen, J.E. Morel, and J.M. McGhee, "Asymptotic Derivation of The Simplified $\mathrm{P}_{\mathrm{N}}$ Equations," Proc. ANS Topical Meeting, Mathematical Methods and Supercomputing in Nuclear Applications, M\&C + SNA '93, April 19-23, 1993, Karlsruhe, Germany, to appear.

10. D. Tomasevic and E.W. Larsen, "The Simplified $\mathrm{P}_{2}$ Correction to the Multidimensional Diffusion Equation," Trans. Am. Nucl. Soc. 66, 32 (1992).

11. Carter, L.L. and Cashwell, E.D., "Particle-Transport Simulation with the Monte Carlo Method," ERDA Critical Review Series, TID-26607, U.S. Department of Energy (Oct. 1975).

12. Lewis, E.E., and Miller, W.F. Jr., Computational Methods of Neutron Transport, Wiley, New York (1984).

13. Los Alamos Monte Carlo Group, "MCNP - A General Monte Carlo Code for Neutron and Photon Transport," LA-7396-M (Rev.), Los Alamos National Laboratory (April 1981).

14. K.N. Abotel, E.W. Larsen, and W.R. Martin, "'Local' Exponential Transform Methods for the Monte Carlo Simulation of Multigroup Transport Problems," Proc. ANS 1992 Topical Meeting on Advances in Reactor Physics, March 8-11, 1992, Charleston 2, 288 (1992). 
15. K.N. Abotel, E.W. Larsen, and W.R. Martin, "'Local' Exponential Transform Methods for the Monte Carlo Simulation of Multigroup, X,Y-Geometry Transport Problems," Proc. ANS Topical Meeting, Mathematical Methods and Supercomputing in Nuclear Applications, $M \& C+S N A$ '93, April 19-23, 1993, Karlsruhe, Germany, to appear.

16. Alcouffe, R.E., "Diffusion Synthetic Acceleration Methods for the Diamond Differenced Discrete-Ordinates Equations," Nucl. Sci. Eng. 64, 344 (1977).

17. E.W. Larsen, "Unconditionally Stable Diffusion-Synthetic Acceleration Methods for the Slab Geometry Discrete-Ordinates Equations. Part I: Theory," Nucl. Sci. Eng. 62, 47 (1982).

18. E.W. Larsen, "Diffusion-Synthetic Acceleration Methods for the Discrete-Ordinates Equations," Proc. ANS Topical Meeting, Advances in Reactor Computations, March 28-31, 1983, Salt Lake City 2, 705 (1983).

19. Larsen, E.W., Morel, J.E., and Miller, W.F., Ir., "Asymptotic Solutions of Numerical Transport Problems in Optically Thick, Diffusive Regimes," J. Comp. Phys. 69, 283 (1987).

20. Larsen, E.W., and Morel, J.E., "Asymptotic Solutions of Numerical Transport Problems In Optically Thick Diffusive Regimes II,"J. Comp. Phys. 83, 212 (1989).

21. Börgers, C., Larsen, E.W., and Adams, M.L., "The Asymptotic Diffusion Limit of a Linear Discontinuous Discretization of a Two-Dimensional Linear Transport Equation," J. Comp. Phys., to appear.

22. E.W. Larsen, "The Asymptotic Diffusion Limit of Discretized Transport Problems," Nucl. Sci. Eng., to appear.

23. Gol'din, V. Ya., "A Quasi-Diffusion Method of Solving the Kinetic Equation," USSR Comp. Math. and Math. Phys. 4, 6, 136 (1967).

24. Larsen, E.W. "Projected Discrete Ordinates Methods for Numerical Transport Problems," Proc. ANS Topical Meeting, International Meeting on Advances in Nuclear Engineering Computational Methods, April 9-11, 1985, Knoxville, Tennessee, 2, 413 (1985). Also published in Nucl. Sci. Eng., 92, 179 (1986).

25. M.M. Miften and E.W. Lursen, "A Symmetrized Quasidiffusion Method for Solving Multidimensional Transport Problems," Trans. Am. Nucl. Soc. 66, 227 (1992). (This is an abridged version of Ref. 26.)

26. M. Miften and E.W. Larsen, "A Symmetrized Quasidiffusion Method for Solving Transport Problems in Multidimensional Geometries," Proc. ANS Topical Meeting, Mathematical Methods and Supercomputing in Nuclear Applications, M\&C + SNA '93, April 19-23, 1993, Karlsruhe, Germany, to appear.

27. R.D. O'Dell, F.W. Brinkley, Jr., and D.R Marr, “User's Manual for TWODANT: A Code Package for Two-Dimensional, Diffusion-Accelerated, Neutral-Particle Transport," LA9814-M, Los Alamos National Laboratory (1982). 
28. C.T. Ballinger, The Response History Monte Carlo Method for Electron Transport, Ph.D. dissertation, Department of Nuclear Engineering, The University of Michigan, Ann Arbor, MI (August 1991).

29. C.T. Ballinger, J.A. Rathkopf, and W.R. Martin, "The Response History Monte Carlo Method for Electron Transport," Nuclear Science and Engineering, 112, 283-295 (1992).

30. C.T. Ballinger, D.E. Cullen, S.T. Perkins, J.A. Rathkopf, W.R. Martin, and S.J. Wildernan, "Single-scatter Monte Carlo compared to condensed history results for low energy electrons," Nuclear Instruments and Methods in Physics Research B72, 19-27 (1992).

31. F. B. Brown and W. R. Martin, "Monte Carlo Methods For Radiation Transport Analysis on Vector Computers", Progress in Nuclear Energy 14, 269(1985).

32. W.R. Martin, "Monte Carlo Methods on Advanced Computer Architectures," Advances in Nuclear Science and Technology 22, 105-164 (1991).

33. W.R. Martin, A. Majumdar, J.A. Rathkopf, and M. Litvin, "Experiences with Different Parallel Programming Paradigms for Monte Carlo Particle Transport Leads to a Portable Toolkit for Parallel Monte Carlo," accepted for presentation at the Joint ANS-ENS International Conference on Mathematical Methods and Supercomputing in Nuclear Applications," Karlsruhe, April 1993. 

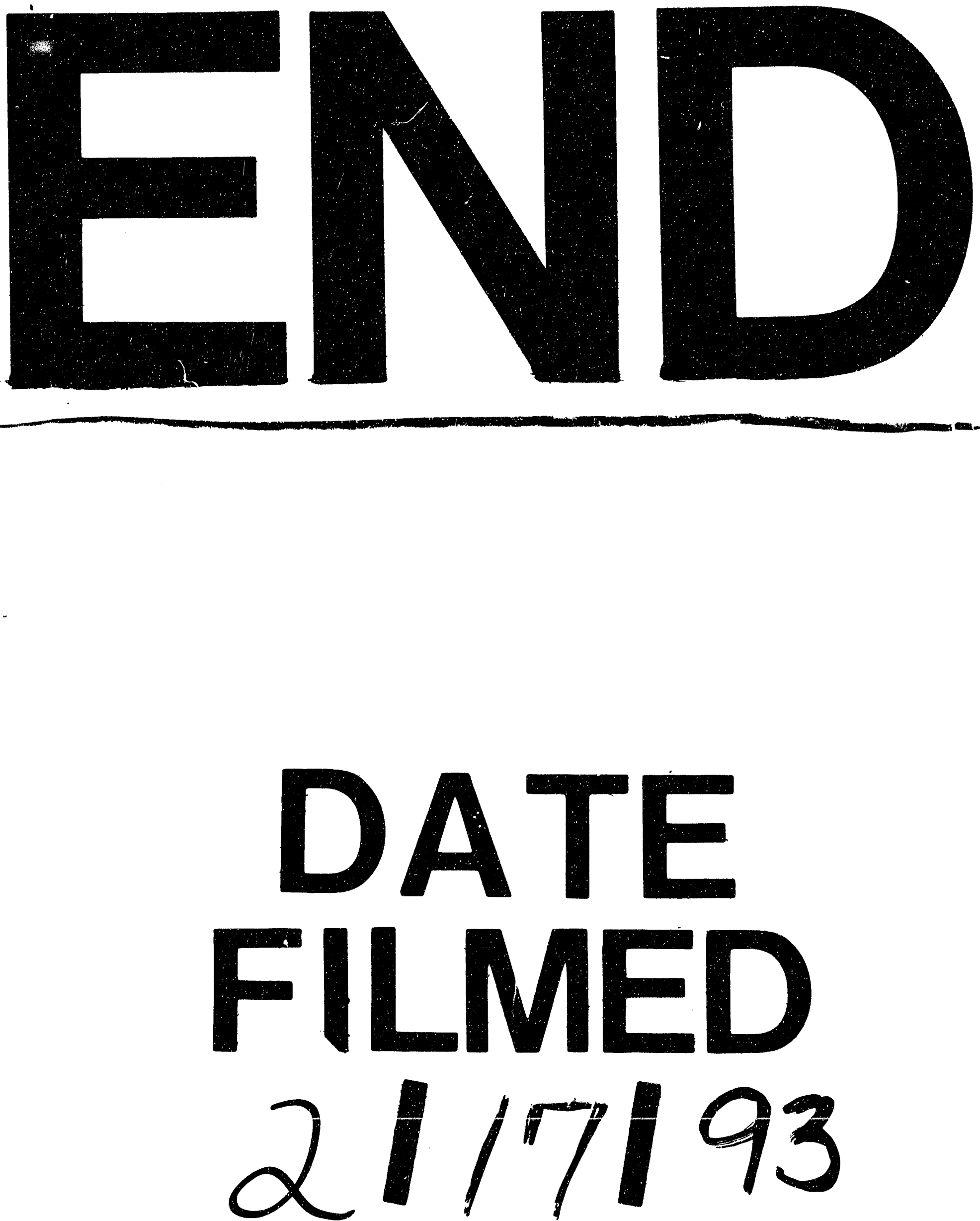


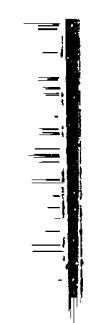

